\title{
Case 2/2019 - Man with Arrhythmogenic Cardiopathy Followed by Rapidly Progressive Heart Failure
}

\author{
Marcella Abunahman Freitas Pereira, ${ }^{\oplus}$ Wilma Noia Ribeiro, ${ }^{\circledR}$ Léa Maria Macruz Ferreira Demarchi ${ }^{\bullet}$ \\ Instituto do Coração (InCor) - Hospital das Clínicas da Faculdade de Medicina da Universidade de São Paulo, São Paulo, SP - Brazil
}

A 36-year-old man was referred to the medical service for surgical treatment of heart failure refractory to drug treatment.

At the age of $26,1^{\text {st }}$-degree atrioventricular block and episodes of non-sustained ventricular tachycardia were detected on the electrocardiogram (ECG). After 4 years, he started to have episodes of pre-syncope.

The magnetic resonance performed at that time (09/29/2010) disclosed diastolic diameter of $59 \mathrm{~mm}$; systolic diameter of $49 \mathrm{~mm}$; 10-mm septum; posterior wall of $11 \mathrm{~mm} ; 48 \%$ left ventricular ejection fraction and $53 \%$ right ventricular ejection fraction, with no contraction abnormalities. The late enhancement imaging showed an infero-septal, medium-basal subepicardial focus, compatible with fibrosis, suggestive of myocarditis or idiopathic dilated cardiomyopathy.

An electrophysiological study was indicated. After extra-stimuli, sustained ventricular tachycardia with hemodynamic instability was triggered and an implantable-cardioverter defibrillator (ICD) was implanted and the patient received a beta-blocker. However, several episodes of ventricular tachycardia were recorded by the ICD and the use of amiodarone was initiated.

He remained asymptomatic for approximately 3 years until he developed heart failure, which rapidly evolved into functional class IV, which resulted in hospitalization for compensation and with acute pulmonary edema at 34 years of age, followed by a new hospitalization a few months later for new heart failure compensation. At that time, hypothyroidism (TSH of 88 um / L) was diagnosed, which was attributed to amiodarone use. The echocardiogram disclosed severe left ventricular systolic dysfunction, with $\mathrm{EF}=21 \%$.

Myocardial resynchronization was indicated, with pacemaker implantation with electrodes implanted at two points in the left ventricle in March 2015, but he was readmitted due to arterial hypotension, atrial fibrillation and heart failure decompensation in September 2015. Amiodarone, dobutamine, spironolactone, furosemide and rivaroxaban were administered.

\section{Keywords}

Heart Failure; Stroke Volume; Cardiomyopathy, Dilated; Myocarditis; Heart Transplantation.

Section Editor: Alfredo José Mansur (ajmansur@incor.usp.br)

Associated editors: Desidério Favarato (dclfavarato@incor.usp.br) Vera Demarchi Aiello (anpvera@incor.usp.br)

Mailing Address: Vera Demarchi Aiello •

Avenida Dr. Enéas de Carvalho Aguiar, 44, subsolo, bloco I, Cerqueira César. Postal Code 05403-000, São Paulo, SP - Brazil

E-mail: demarchi@cardiol.br, anpvera@incor.usp.br

DOI: $10.5935 / a b c .20190065$
The patient was transferred to the Heart Institute (InCor) for possible surgical treatment of heart failure (heart transplantation) on October 20, 2015. The patient reported a $20 \mathrm{~kg}$ loss over a 4-year period. He denied arterial hypertension and diabetes mellitus.

Physical examination showed cachexia, jugular swelling + , hepatojugular reflux, no distension alteration at the Valsalva maneuver or exhalation, vesicular murmurs present in the lungs, slightly reduced on the left pulmonary basis, palpable thrill in the mitral, tricuspid, accessory aortic foci; arrhythmic heart sounds with a more audible holosystolic murmur in the mitral focus, radiating into the posterior axillary line. The abdomen was flat, with a $6-\mathrm{cm}$ hepatomegaly from the right costal border, palpable caudate lobe nearby, without ascites. Lower limbs without edema, with no signs of deep venous thrombosis. He was receiving intravenous dobutamine.

The patient was evaluated by the heart transplant team due to the persistent need of high-dose inotropic and vasodilator drugs during ICU observation. The patient was prioritized for cardiac transplantation due to use of vasoactive drug and joined the list on 11/09/15.

The ECG showed a pacemaker rhythm operating in VAT mode and chest X-ray showed cardiomegaly with signs of pulmonary congestion.

Laboratory tests (10/21/2015) showed hemoglobin $11.1 \mathrm{~g} / \mathrm{dL}$, hematocrit 34\%, leukocytes 7290 (neutrophils 82\%, eosinophils $3 \%$, lymphocytes $7 \%$, monocytes $6 \%$ ), platelets $259,000 / \mathrm{mm}^{3}$, urea $28 \mathrm{mg} / \mathrm{dL}$, creatinine $0.92 \mathrm{mg} / \mathrm{dL}, \mathrm{CRP}$ of $69.34 \mathrm{mg} / \mathrm{L}$, sodium $137 \mathrm{mEq} / \mathrm{L}$, potassium $3.4 \mathrm{mEq} / \mathrm{L}$, PAT (INR) of 2.4; APTT (rel. Times) of 1.31; Urinalysis with proteinuria of $0.67 \mathrm{~g} / \mathrm{L}$.

The echocardiogram (10/21/2015) disclosed left ventricle with diffuse hypokinesia, worse in the inferior and inferolateral walls and ejection fraction of $25 \%$; the right ventricle showed moderate diffuse hypokinesia. There was marked mitral regurgitation; the other valves showed no alterations. The pulmonary artery pressure was estimated at $49 \mathrm{mmHg}$.

He had two bloodstream infections, which were treated with meropenem and vancomycin and tazobactam during the month of November 2015.

Serological tests were positive for toxoplasmosis and mononucleosis in the IgG.

Abdominal, thyroid and carotid artery ultrasonography results were normal.

Right-chamber catheterization disclosed systolic pulmonary artery pressure of $55 \mathrm{mmHg}$, diastolic pressure of $23 \mathrm{mmHg}$ and a mean pressure of $34 \mathrm{mmHg}$; the pulmonary occlusion pressure was $24 \mathrm{mmHg}$, the cardiac output was $5.5 \mathrm{~L} / \mathrm{min}$ and the transpulmonary gradient was $10 \mathrm{mmHg}$; pulmonary 
vascular resistance was 1.8 Woods and the systemic vascular resistance was 887 dynes $/ \mathrm{sec} / \mathrm{cm}^{-5}$.

The transplantation was performed in March 2016 using the bicaval orthotopic heart transplantation technique, without complications; the patient received prophylactic antimicrobial treatment with vancomycin and cefepime.

After the transplantation, the immunosuppressant drugs prednisone, cyclosporine and mycophenolate were introduced. Endomyocardial biopsies performed on March 21 and 31 showed grade I rejection and cytomegalovirus tests were negative.

The echocardiogram performed at hospital discharge on 03/28/2016 showed left atrium of $42 \mathrm{~mm}$, septum and posterior wall of $11 \mathrm{~mm}$, left ventricle of $50 \times 31$ with ejection fraction of $68 \%$; normal right ventricle and pulmonary artery pressure of $35 \mathrm{mmHg}$.

The medication prescribed at the hospital discharge consisted of cyclosporin 100mg $+75 \mathrm{mg}$ daily, prednisone 40mg 1x / day, mycophenolate sodium 720mg every $12 \mathrm{~h}$.

At the outpatient clinic consultations he remained asymptomatic (April 2017). He currently takes Tacrolimus $4 \mathrm{mg}$

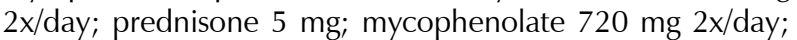
diltiazem $30 \mathrm{mg} 3 \mathrm{x} /$ day; simvastatin $10 \mathrm{mg}$ 1x/day; vitamin D $900 \mathrm{mg} /$ day; and omeprazole 20mg 1 x/day.

\section{Clinical aspects}

The patient developed arrhythmia at 26 years of age. At age 33, in 2013, he developed heart failure, which rapidly progressed to functional class IV, with consecutive hospitalizations due to acute decompensation. In 2015, after the last hospitalization, he was placed on the priority list for heart transplantation due to clinical treatment refractoriness. In March 2016 he underwent the procedure and remained asymptomatic, being followed through outpatient clinic consultations since April 2017.

This is a heart failure case with important aspects that must be investigated: etiology and factors for decompensation.

It is suggested that the probable cause of the index event is myocarditis or idiopathic dilated cardiomyopathy in a 30-year-old man.

The American Heart Association classifies primary cardiomyopathies (predominant heart involvement) into three groups: genetic (hypertrophic cardiomyopathy, right ventricular arrhythmogenic cardiomyopathy, noncompacted left ventricle, glycogen accumulation disease, mitochondrial myopathies and channelopathies); mixed, predominantly non-genetic (dilated cardiomyopathy, restrictive); and acquired (inflammatory (myocarditis), caused by stress (Takotsubo), peripartum, induced by tachycardia, and of the infant, child of an insulin-dependent mother. ${ }^{1}$

The origin of inflammatory heart diseases can be: autoimmune (connective tissue diseases, sarcoidosis, eosinophilic diseases); inflammatory diseases (hypersensitivity myocarditis, endomyocardial fibrosis, hypereosinophilic syndrome); toxic (antineoplastic chemotherapeutic drugs); and infectious (protozoa, fungi, bacteria, viruses and parasites). ${ }^{2}$
Among the infiltrative diseases, which usually occur simultaneously with restrictive syndrome, are: amyloidosis, sarcoidosis and deposition diseases (Fabry, and others). ${ }^{3}$

Amyloidosis could be the etiology of the patient's heart disease, since it is a progressive disease, affects adults from the age of 30 years and its frequent form of extracardiac involvement is kidney disease. In this context, the patient was close to 30 years old and his urinalysis showed the presence of proteinuria. Additionally, the ECG in amyloidosis usually shows atrioventricular block, supraventricular and ventricular arrhythmias, as the patient showed at the beginning of the clinical picture. However, the typical findings at the magnetic resonance and echocardiogram show an enlargement of the septum and posterior wall, which were absent in the present case.

In sarcoidosis, there is a more frequent involvement of individuals between 25 and 60 years, and it is positively associated with lung and lymph node involvement and frequent extracardiac alterations, which were absent in the patient.

Fabry disease, on the other hand, manifests in childhood or adolescence, and shows important dermatological findings, which rule out the possibility of this diagnosis. Regarding the complementary exams, amyloidosis and Fabry's disease show findings that are similar to sarcoidosis on the ECG, magnetic resonance imaging and echocardiogram..$^{4,5}$

However, there are no reports of autoimmune tests, extracardiac investigations, much less the performance of endomyocardial biopsy in the current patient with rapidly progressive heart failure, without a definitive cause, not responsive to clinical treatment and with hemodynamic deterioration. ${ }^{5}$

Recreational drug poisoning, such as alcohol, amphetamines, cocaine and the use of anabolic drugs could be possible causes for myocarditis, considering that the patient is young and a potential user of these drugs. However, the current case does not show a history of drug addiction or drug abuse. Moreover, drug poisoning is expressed by chamber dilatation and not by myocardial thickening.

In South America and Brazil, the Chagasic etiology is a frequent form of myocarditis. However, the patient did not have alterations in the ECG and echocardiogram suggestive of this disease. There was no evidence of right bundle-branch block, anterosuperior divisional block, apical aneurysm, right heart failure manifestations, and the patient did not show positive epidemiology for Chagas disease..$^{5-7}$

Viruses are also frequent infectious myocarditis agents, with the most common agents being adenovirus, enterovirus, parvovirus, herpes simplex, hepatitis $\mathrm{C}$ virus, cytomegalovirus and Epstein-Barr virus. However, magnetic resonance imaging shows thickening of the septum and posterior wall, which indicates other causes of myocarditis, since the infectious one is associated with dilated cardiomyopathy. ${ }^{8}$

However, there was not a complete investigation to elucidate a possible infectious cause, either by serology or by endomyocardial biopsy of the right ventricle. The patient is young, viral contaminations are common, he may have circulated in areas of greater risk for Chagas disease contamination and be sexually active, increasing the chances of being infected with HIV and different types of hepatitis. ${ }^{8}$ 
Regarding the less probable diagnostic hypothesis, one must consider coronary artery disease. Despite presenting segmental dysfunction at the MRI, we have here a young patient with no clinical features or risk factors for this etiology. Additionally, the thickening of the septum and posterior wall have systemic arterial hypertension and hypertrophic cardiomyopathy as important differential diagnoses. However, the patient did not suffer from arterial hypertension and the echocardiography, as well as the magnetic resonance imaging, did not show any characteristic findings of hypertrophic cardiomyopathy: asymmetric septal hypertrophy, left ventricular outflow tract obstruction and septum/wall ratio $>1.3$. Valvular disease can also be ruled out, since the patient did not show it initially, either at the physical examination or imaging test, and the patient only developed mitral regurgitation after heart failure progression.

Finally, idiopathic dilated cardiomyopathy cannot be ruled out as a diagnostic possibility for this case. It typically affects men between the ages of 18 and 50 and at least $25 \%$ of the cases show genetic transmission of the disease. It is believed that genetic factors associated with changes in immune response and infectious factors could act synergistically in the development of structural changes and consequent onset of clinical manifestations. It is estimated that between 10\%-20\% of cases of idiopathic cardiomyopathy are caused by a previous viral infection sequela. ${ }^{8,9}$

The patient rapidly evolved to functional class IV, showing marked ejection fraction reduction and underwent successive hospital admissions due to the decompensations.

There are many factors that exacerbate heart failure and taking into account the patient's history, one cannot infer a specific precipitating factor for the case described herein. Among the possible hypotheses is the natural evolution of the underlying disease, of which etiology was not clarified, and this fact may have prevented the implementation of specific treatment strategies.

The following are other possible precipitating factors of the acute decompensation observed in the patient: ${ }^{3,5,10,11}$ absence of health education performed by the professionals and/or the patient's poor adherence to non-pharmacological measures for heart failure management; inadequate diet and water intake, as well as the abuse of alcohol and other drugs, are frequent factors for decompensation. Moreover, all patients with heart failure should be vaccinated against influenza and pneumococcus, considering that respiratory infections are common etiologies for decompensation; however, in this case, the patient showed no signs of infection leading to hospitalization or leukogram alterations.

As for pharmacological measures, the use of beta-blockers (carvedilol, nebivolol, bisoprolol and metoprolol succinate) in patients with reduced ejection fraction associated with angiotensin-converting enzyme inhibitor is the effective treatment for patients with New York Heart Association functional class I to IV, because they reduce morbimortality by acting on cardiac reverse remodeling. There are contraindications for the use of these classes of drugs; however, there are no records in the clinical case of reasons justifying the absence of introduction of these classes of drugs after the development of systolic heart failure. Furthermore, systolic insufficiency refractory to optimized clinical treatment and ejection fraction $\leq 35 \%$, also requires the use of aldosterone antagonists, a medication that also has an effect on reverse remodeling, if the patient does not have contraindications to its use. There is information about the introduction of this drug only after the third decompensation event.

Arrhythmia, in turn, is an important decompensatory factor, such as the atrial fibrillation developed by this patient during one of the hospitalizations. Its onset is associated with several adverse hemodynamic effects, such as loss of atrioventricular synchrony and loss of atrial contraction, leading to cardiac output reduction in a heart with an already impaired ventricular function.

Other possible etiologies for decompensation detected in this patient were the concomitant presence of anemia and kidney dysfunction, which are conditions that considerably increase mortality in heart failure.

The patient's hemoglobin level was $11.1 \mathrm{~g} / \mathrm{dL}$. Being an important precipitating factor, anemia becomes important due to its deleterious effects on the heart. The erythrocytes, in addition to providing oxygen to myocardial cells, favor the exchange of antioxidants that prevent oxidative stress and programmed cell death, but the impairment of these mechanisms favor myocardial dysfunction. Additionally, in response to hypoxemia resulting from anemia, the sympathetic system is stimulated, leading to tachycardia, increased inotropism and vasoconstriction, further compromising myocyte function and leading to hypervolemia in parallel. Specifically, hypoxemia of anemia and kidney vasoconstriction generate renal ischemia, with the release of inflammatory factors related to myocardial injury and hypervolemia due to the activation of the renin-angiotensin-aldosterone system.

Proteinuria was observed in a urinalysis result, despite normal values of creatinine and urea. Like anemia, nephropathy may be a factor of decompensation, etiology and/or consequence of heart failure. As a precipitating factor, one can point to salt and water retention; alterations in the cardiomyocyte structure and function due to inflammatory activation and calcium and phosphorus metabolism abnormalities; and due to the anemia itself, generated by kidney dysfunction, leading to reduced erythropoietin production. One should consider that nephropathy can also be a consequence of amyloidosis, as it leads to amyloid deposition in the kidneys, impairing their function, and $80 \%$ of patients with this disease have proteinuria.

Due to increased pulmonary artery and right ventricular systolic pressure, pulmonary embolism could be a cause for decompensation. However, in this clinical setting, these cardiopulmonary alterations are possibly due to heart failure progression.

Hypothyroidism is a potential cause of heart failure due to bradycardia, systolic and diastolic dysfunction, increased systemic vascular resistance, diastolic hypertension, increased arterial stiffness and endothelial dysfunction. ${ }^{12}$ Laboratory examination showed the patient had TSH of $88 \mathrm{um} / \mathrm{L}$ and the introduction of levothyroxine was not reported.

The patient may also have decompensated due to marked mitral regurgitation caused by left ventricular 
dilatation and, in this situation, valvulopathy was secondary to the disease progression.

The use of negative inotropic medications, corticosteroids, cardiotoxic chemotherapeutic drugs, non-steroidal anti-inflammatory drugs, antiarrhythmics and glitazone or dipeptidyl phosphatase 4 inhibitors may be decompensation triggers, which can be used through self-medication or by not informing to other physicians about the presence of heart failure. (Dr. Marcella Abunahman Freitas Pereira, Dr. Wilma Noia Ribeiro)

Diagnostic hypotheses: Infiltrative cardiomyopathy or idiopathic dilated cardiomyopathy. (Dr. Marcella Abunahman Freitas Pereira, Dr. Wilma Noia Ribeiro)

\section{Anatomopathological examination}

The explanted heart, devoid of the atria, weighed $598 \mathrm{~g}$ (normal $=300$ to $350 \mathrm{~g}$ ). Externally, there was discrete and focal epicardial thickening on the sternocostal and diaphragmatic surfaces of the right and left ventricles. The cross-sectional sections showed a bicuspid aortic valve, with fusion between the left semilunar and non-coronary leaflets, without a median raphe (Figure 1), moderate and diffuse thickening of the semilunar leaflets, with marked retraction and slight calcification at the free border of the semilunar leaflets, macroscopically suggestive of valve regurgitation. The right coronary artery (RCA) ostium was typically located in the right Valsalva sinus. In the left Valsalva sinus, two ostia of coronary arteries originated: the most anterior ostium gave origin to the circumflex (Cx) artery and the posterior ostium, tangentially, originated the anterior descending artery (ADA) (Figure 1). The proximal segments of the ADA and $\mathrm{Cx}$ intersected, with the ADA being positioned higher than the $\mathrm{Cx}$ (Figure 2). An intramyocardial ("myocardial bridge") trajectory from the fifth to the seventh centimeter of the ADA was also observed (Figure 3 ). Right predominant coronary artery circulation was observed. There was no significant luminal obstruction in the coronary ostia or epicardial coronary arteries. There was moderate ventricular hypertrophy and dilatation and moderate atrial dilatation. Presence of moderate and diffuse retraction at the free border of the anterior cusp of the mitral valve was observed. The tricuspid and pulmonary valves showed discrete and diffuse retraction at the free border of their leaflets. Emerging from the superior vena cava, there was a cardiac pacemaker cable-lead that was implanted in the endocardium of the anterior wall of the right atrium. Another cardiac pacemaker cable-lead extended from the superior vena cava through the right atrium, tricuspid valve and right ventricle, and was implanted in the ventricular septum endocardium, in its apical portion. There were no thrombi in the heart cavities. The histological study showed moderate hypertrophy in cardiomyocytes and diffuse interstitial myocardial fibrosis, more pronounced in the left ventricle (Figure 4). (Dr. Léa Maria Macruz

\section{Ferreira Demarchi)}

Anatomopathological diagnoses: 1) bicuspid aortic valve; 2) Ventricular hypertrophy and dilatation; 3) Diffuse interstitial myocardial fibrosis, more pronounced in the left ventricle; 4) Congenital anomalies of the origin, course of the anterior and circumflex interventricular epicardial coronary arteries. (Dr. Léa Maria Macruz Ferreira Demarchi)

\section{Comment}

An association was observed between the bicuspid aortic valve and anatomical alterations in the coronary arteries in the patient's explanted heart, which is a frequent

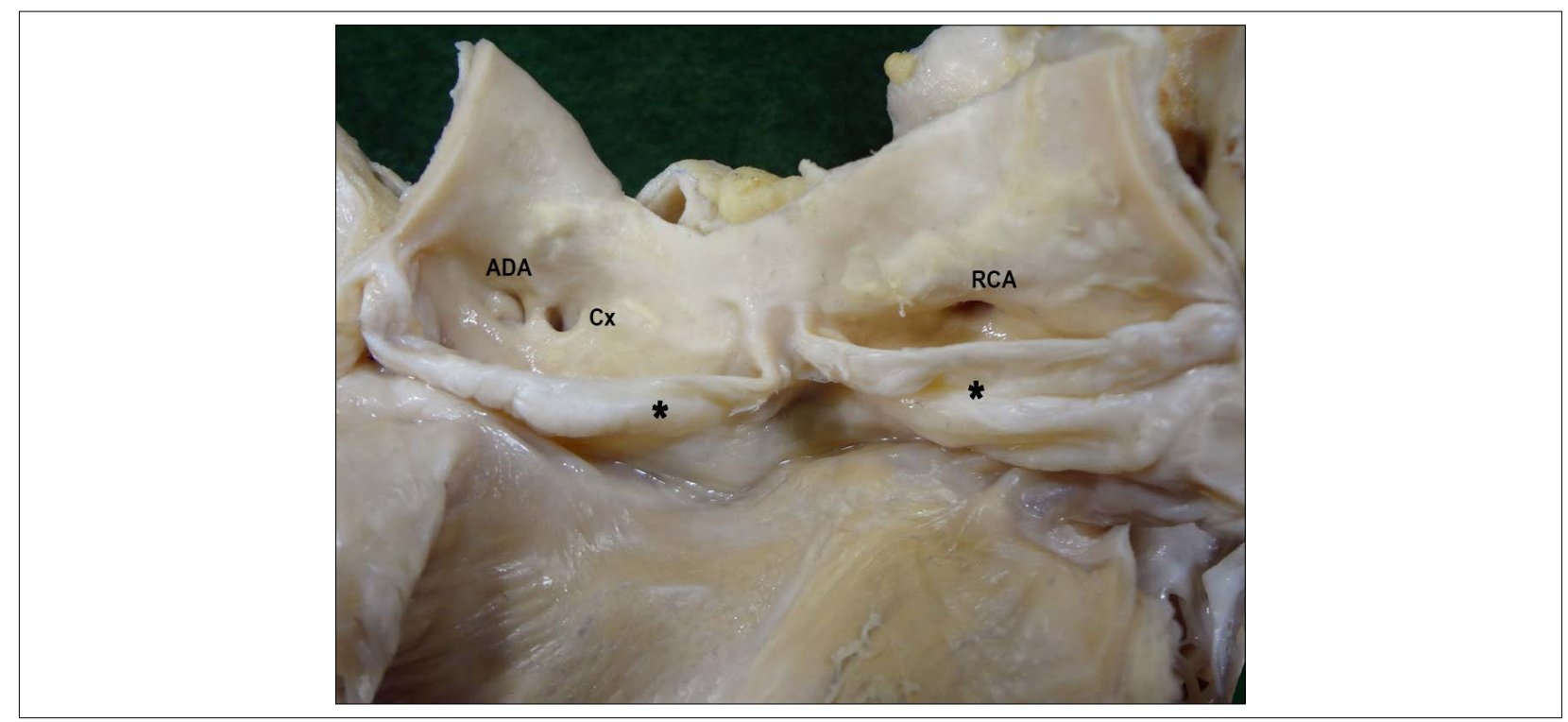

Figure 1 - Left ventricular outflow tract: bivalve aortic valve, showing thickened semilunar leaflets $\left({ }^{*}\right)$, with moderate retraction at the free border, suggestive of valvular regurgitation. The anterior descending $(A D A)$ and circumflex $(C x)$ coronary arteries originate from separate ostia, in the left Valsalva sinus; the ADA ostium lies posterior to the $\mathrm{Cx}$ ostium and is tangential. The right coronary artery (RCA) ostium is located in the right Valsalva sinus. 


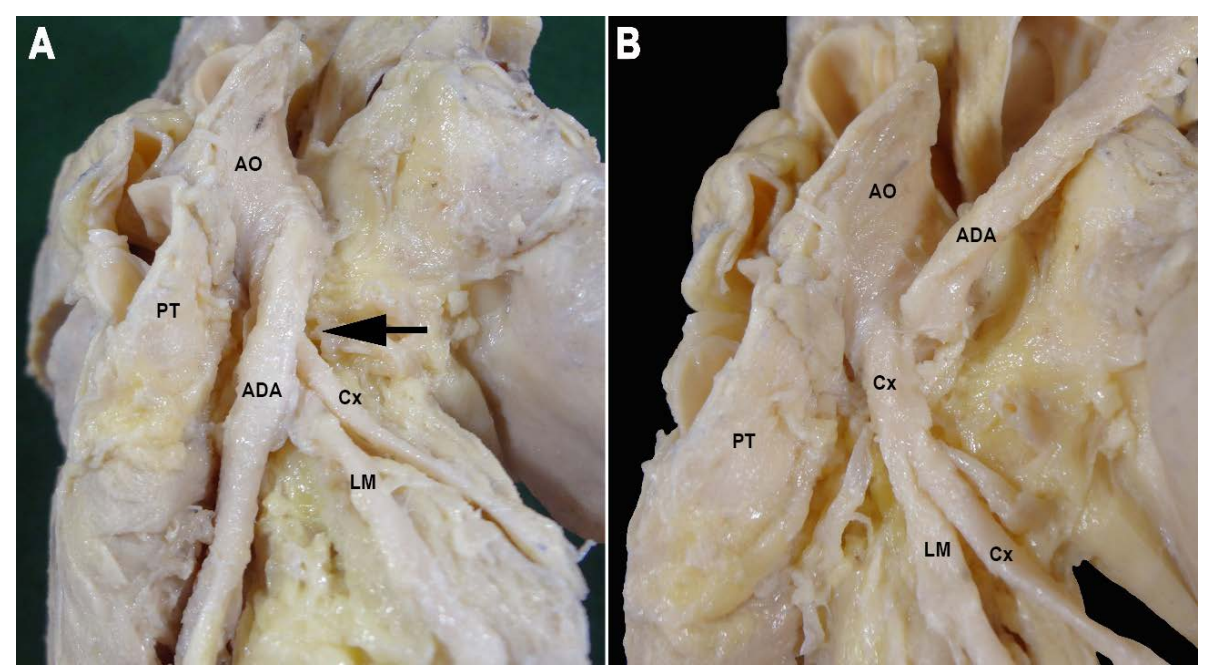

Figure 2 - Left lateral surface of the base of the heart: A) Crossing (arrow) of the proximal epicardial segments of the anterior descending artery (ADA) and circumflex (Cx) artery; the ADA is in a position above the $C x . B) C x$ course from its origin at the aorta ( $A O)$. The ADA has been folded superiorly to show the circumflex artery epicardial course. LM: left marginal branch of the CX. PT: pulmonary trunk.

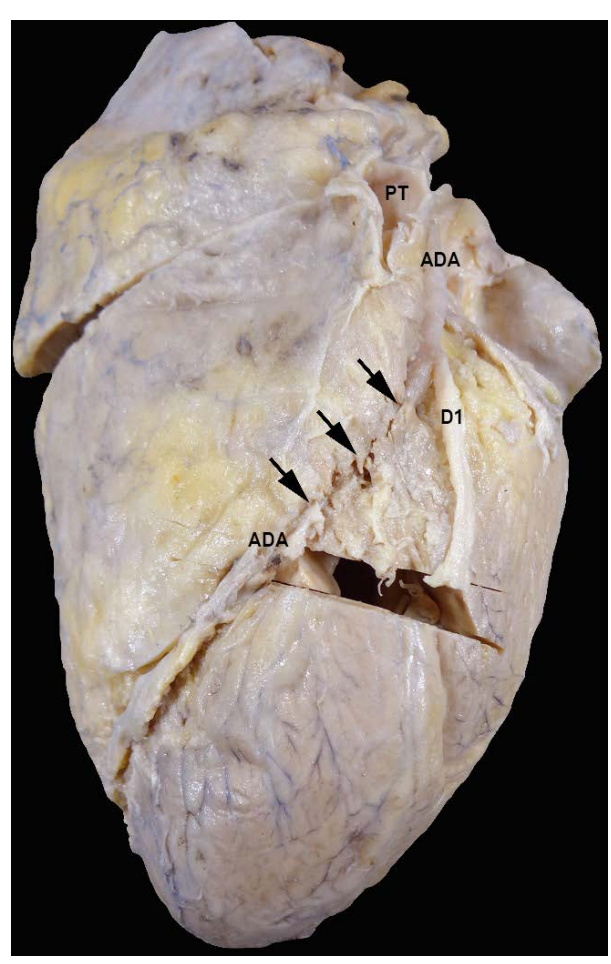

Figure 3 - Sternocostal surface of the heart: intramyocardial course (arrows) of the anterior descending artery (ADA). D1- First diagonal branch of the ADA. PT: pulmonary trunk.

finding in the literature..$^{13}$ The bicuspid aortic valve is the most prevalent cardiac congenital anomaly and, in autopsy studies, its incidence ranges from $0.9-2.5 \%$ in the general population, ${ }^{14}$ being more frequent in male individuals, with a men/women ratio ranging from 1.8 to $5.6 .{ }^{15}$ The anatomical alterations in coronary arteries may represent variations of the normal anatomy or congenital anomalies, depending on their incidence in the general population. ${ }^{16}$ Alterations in the general population are known as variants or anatomical variations of the normal in the general population, whereas those occurring in less than $1 \%$ are defined as congenital anomalies. The incidence of coronary anomalies ranges from $0.2 \%$ to $1.2 \%$ in the different series found in the literature, depending on the analyzed population and the methods 


\section{Anatomopathological Correlation}

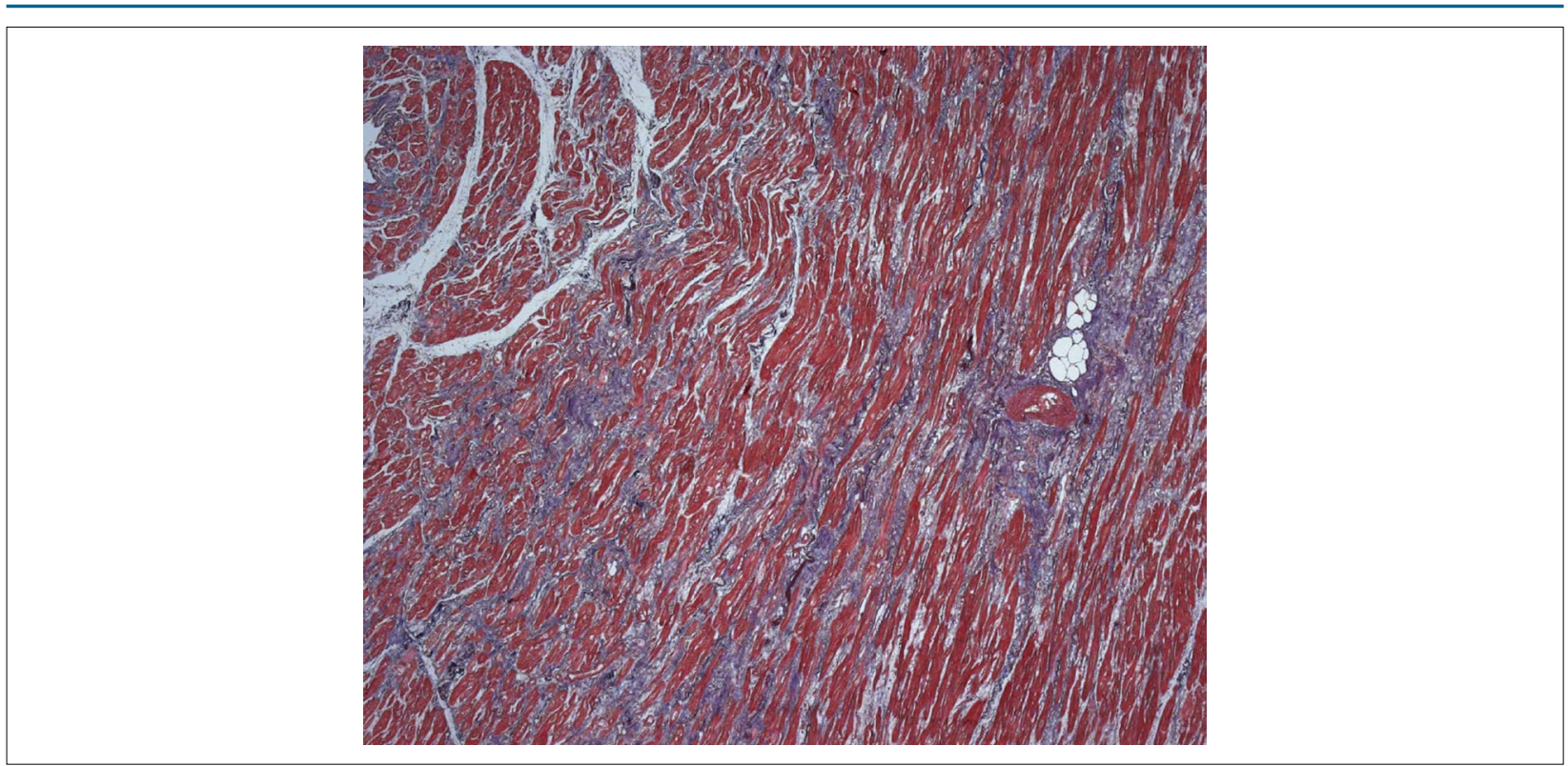

Figure 4 - Photomicrography of the left ventricular myocardium: Diffuse myocardial fibrosis (in blue). Masson's trichrome, 50x).

used. ${ }^{17}$ Anatomopathological ${ }^{18}$ and coronary angiography studies ${ }^{19}$ performed in hearts of individuals without other congenital heart malformations divide the coronary anomalies into two groups: those of anomalous arterial origin and course and intrinsic anatomical anomalies of the arteries. In the case of this patient, we observed anomalous origin of the coronary arteries, represented by the absence of the left coronary artery and independent origin in separate ostia and in the same sinus of Valsalva of the ADA and the circumflex artery. The intramyocardial course of the ADA is classified as an anatomical variation, since its occurrence in the middle segment of the ADA ranges from $5 \%$ to $80 \%$ of patients in different studies. ${ }^{20}$ In the other coronary arteries, such an alteration is considered an anomaly, since it occurs is less than $1 \%$ of the population. The crossing of epicardial branches is an anomaly of the intrinsic anatomy of the coronary arteries and is quite rare, with few cases having been described in the literature..$^{20,21}$ One might question whether there was compression of the arterial segments involved in the epicardial crossing, but in the absence of obstructive coronary alterations and localized ischemic myocardial lesions, anatomopathological examination is limited for such evaluation. Left ventricular hypertrophy and dilatation, as well as diffuse interstitial myocardial fibrosis can be explained by the bicuspid aortic valve dysfunction. (Dr. Lea Maria Macruz Ferreira Demarchi)

\section{References}

1. McKenna WJ, Maron BJ, Thiene G. Classification, epidemiology, and global burden of cardiomyopathies. Circ Res. 2017;121(7):722-30

2. Trachtenberg BH, Hare M. Inflammatory cardiomyopathic syndromes. Circ Res. 2017;121(7):3-18.

3. Muchtar E, Blauwet LA, Gertz MA. Restrictive cardiomyopathy. Genetic, pathogenesis, clinical manifestations, diagnosis, and therapy. Cir Res. 2017;121(7):819-37

4. Seward J.B., Casaclang-Verzosa G. Infiltrative cardiovascular diseases: cardiomyopathies that look alike. J Am Coll Cardiol. 2010;55(17):1769-79.

5. Dickstein K, Cohen-Solal A, Filippatos G, McMurray JJ, Ponikowski P, PooleWilson PA, et al. ESC Guidelines for the diagnosis and treatment of acute and chronic heart failure 2008: the Task Force for the Diagnosis and Treatment of Acute and Chronic Heart Failure 2008 of the European Society of Cardiology. Developed in collaboration with the Heart Failure Association of the ESC (HFA) and endorsed by the European Society of Intensive Care Medicine (ESICM). Eur Heart J. 2008;29(19):2388-442.
6. Dias JCD, Ramos Jr AN, Gontijo ED, Luquetti A, Shikanai-Yasuda MA, Coura JR, et al. II Consenso Brasileiro em Doença de Chagas,2015. Epidemiol Serv Saude.2016;25(num esp):7-86.

7. Maisch B, Ristic AD, Seferovic P.M. New directions in diagnosis and treatment of pericardial disease: a project of the Taskforce on Pericardial Disease of the World Heart Federation. Herz. 2000;25(8):769-80.

8. Babonian C, Treasure T. Meta-analysis of the association of enteroviroses with human heart disease. Heart. 1997;78(6):539-43.

9. McNally EM, Mestroni L. Dilated cardiomyopathy . Genetic determinants and mechanisms. Circ Res. 2017;121(7):731-48.

10. Ponikowski P, Voors AA, Anker SD, Bueno H, Cleland JGF, Coats AJS, Falk V. Guidelines for the diagnosis and treatment of acute and chronic heart failure: The Task Force for the diagnosis and treatment of acute and chronic heart failure of the European Society of Cardiology. Eur Heart J. 2016;37(27):2129-200. 
11. Ahmad A, Rand W, Manjunath J. Reduced kidney function and anemia as risk factors for mortality in patients with left ventricular dysfunction. J Am Coll Cardiol. 2001;38(4):955-62.

12. Biondi B. Heart failure and thyroid function. Eur J Endocrinol. 2012;167(5):609-18.

13. Angelini P, Villason S, Chan AV Jr, Diez JG. Normal and anomalous coronary arteries in humans. In: Angelini P (ed). Coronary artery anomalies: a comprehensive approach. Philadelphia: Lippincott Williams \& Wilkins; 1999. p. 27-150.

14. Roberts WC. The congenitally bicuspid aortic valve. A study of 85 autopsy cases. Am J Cardiol. 1970;26(1):72-83.

15. Subramanian R, Olson LJ, Edwards WD. Surgical pathology of pure aortic stenosis: a study of 374 cases. Mayo Clin Proc. 1984;59(10):683-90.
16. Angelini P. Normal and anomalous coronary arteries: definitions and classification. Am Heart J. 1989;117(2):418-34

17. Burke A, Tavora F (eds). Practical cardiovascular pathology: nonatherosclerotic coronary artery disease. Philadelphia: Wolters Kluwer Health / Lippincott Williams \& Wilkins; 2011. p.118-39.

18. Roberts WC. Major anomalies of coronary arterial origin seen in adulthood Am Heart J. 1986;111(5):941-63.

19. Angelini P, Trivellato M, Donis J, Leachman RL. Myocardial bridges: a review. Prog Cardiovasc Dis. 1983;26(1):75-88.

20. Continentino MA, Freitas AM. Crossing coronary arteries. Arq Bras Cardiol. 2011;96(4):e76.

21. Andreou AY, Kyprianou D, Eteocleous N, Theodorou S, Avraamides PC. A case of crossing coronary arteries. J Cardiovasc Med. 2012;13(5):332-3. 